\title{
Ueber das Verhältniss von Nerve und Muskel.
}

\author{
Von
}

P. Tergast, Cand. med. in Göttingen.

Hierzu Tafel II.

Anatomie und Physiologie sind in gleicher Weise an der Lösung der Frage interessirt, wie sich der eintretende Nervenstamm zum ganzen Muskel verhält, und doch sind die darüber vorliegenden Arbeiten äusserst spärlich. Meist findet man die Beobachtungen mehr zufällig, gelegentlich anderer Untersuchungen gemacht. Es erklärt sich dies leicht durch das Verlangen, Klarheit über die letzten Endigungen des motorischen Nerven, den Zusammenhang mit der Muskelfaser selbst zu verbreiten, ohne sich um weiter Zurückliegendes zu bekümmern. Da nun aber auch dies letztere nicht ohne Wichtigkeit zu sein schien, so wurden die hier mitgetheilten Untersuchungen gemacht, die mit Hilfe einer exacten Methode vollkommen sichere Resultate ergaben.

Eigentlich die einzige Abhandlung, welche das Verhältniss zwischen Nerv und Muskel zum Gegenstande hat, ist die von Reichert ${ }^{1}$ ), der den Brusthautmuskel des Frosches studirte und zu dem merkwürdigen Resultate kam, dass in diesem aus 160 Fasern bestehenden Muskel nur 6-7 Nervenröhren treten, die dann innerhalb des Muskels selbst, durch Theilung in die erforderliche Anzahl von Zweigen zerfallen. Durch die gelegentlichen Bemerkungen späterer Autoren bildete sich dann allmälig die Ansicht heraus,

1) Müller's Archiv, 1851. 
wie sie z. B. Kühne ${ }^{1}$ ) und Frey ${ }^{2}$ ) aussprechen, dass mit der Höhe der Organisation der verschiedenen Wirbelthiere die Zahl der in den Muskel eintretenden Nerven zunehme.

Eine Prüfung dieser Angaben musste nun die Aufmerksamkeit zuerst auf die Augenmuskeln lenken, welche allein in der ganzen Wirbelthierreihe ein constantes Verhältniss bieten sollten ( $\mathrm{K} u ̈ h \mathrm{he}$ 1. c.).

Die bei Untersuchung derselben eingeschlagene Methode, welche nur absolut sichere Resultate ergeben konnte, war die, durch Zählung von Querschnitten des Muskels sowohl, wie des Nerven in Ziffern ausdrückbare Werthe zu bekommen. Zu diesem Behuf musste jedoch erst die wichtige Vorfrage erledigt werden, ob die Fibrillenbündel den ganzen Muskel durchlaufen, oder ob sie nur einen Theil desselben durchsetzen; nur wenn ersteres der Fall war, was nach Krause's Angaben ${ }^{3}$ ) wahrscheinlich sein musste, genügte ein einfacher Querschnitt in der Mitte des Muskels, um genau die Zahl der in ihm enthaltenen Fasern eruiren zu können. Kühne ${ }^{4}$ ) hat nach Budge's Vorgang ein brauchbares Hülfsmittel zu Gebote gestellt, die Muskelfäden in isolirtem Zustand zu untersuchen, indem nach seiner Anweisung der Muskel in einem Gemisch von Crystallen des chlorsauren Kali und concentrirter Salpetersäure vollständig vergraben nach einiger Zeit so macerirt wird, dass die einzelnen Fibrillenbündel, besonders durch Schütteln in einem Probirröhrchen, ohne Verletzung auseinanderfallen.

Wirklich zeigte sich nun auch, dass an den Augenmuskeln von Schafen, die vorwiegend zur Untersuchung benutzt wurden, die Muskelfasern zum grössten Theil die Länge des ganzen Muskels einnehmen. Eine Anzahl derselben endete auch etwas früher, doch liess sich jedenfalls soviel mit voller Sicherheit bestimmen, dass ein Querschnitt, durch die Mitte des Muskels gelegt, sämmtliche Fasern enthalten muss. Es ergab sich nun aber der merkwürdige Umstand, dass ein solcher Querschnitt anscheinend sogar mehr Fa-

1) Handb. d. Lehre v. d. Geweben, von Stricker. Bd. I. 1868, p. 148.

2) Histologie, 3. Aufl. p. 321,

3) W. Krause: Motorische Endplatten. Hannover 1869.

4) Peripher. Endorgane d. mot. Nerven. Leipzig 1862. 
sern enthält, als in Wirklichkeit vorhanden sind, indem diese sich nicht selten theilen ${ }^{1}$ ).

Es wäre nun natürlich zur richtigen Schlussfolgerung erforderlich, diese Pseudofasern nach ungefährer Schätzung vou der Gesammtzahl der Fasern in Abzug zu bringen, doch habe ich geglaubt, davon Abstand nehmen zu können, einmal weil die Zahl der Abzweigungen, die auf einem solchen Querschnitte vorkommen, eine so minimale genannt werden muss, dass ihre Anwesenheit auf die Beziehungen der Gesammtzahl der Muskelfäden zu den Nervenröhren nicht den geringsten Einfluss auszuüben im Stande ist, und dann, weil ja diese Abzweigungen ebenfalls mit Nerven versorgt werden, und schon deshalb als selbständige Fasern betrachtet werden können.

Nachdem also erwiesen, dass ein Querschnitt genau durch die Mitte des Muskels gefiuhrt, einen fast vollständig richtigen Nachweis der in demselben enthaltenen Fasern geben muss, wende ich mich zu den Zahlenverhältnissen, die sich bei den Untersuchungen herausgestellt haben. Die zur Beobachtung verwendeten Muskeln wurden möglichst von dem äusserlich haftenden Fett und Bindegewebe rein präparirt und dann mit dem zugehörigen Nerven in absolutem Alkohol gehärtet. Zur schärferen Abgrenzung der Conturen der einzelnen Muskelfasern liess ich die Schnitte einige Zeit in Carmin sich färben und schritt erst dann zur eigentlichen Zählung. Dasselbe Verfahren wurde bei der Behandlung des Nerven eingeschlagen, und zwar wurde hier der Schnitt möglichst nahe an der Eintrittsstelle in den Muskel geführt. Selbstverständlich können hier unter dem eintretenden Nerven nur diejenigen Nervenröhren verstanden sein, welche sich ausschliesslich auf den betreffenden Muskel beschränken, nicht etwa auch solche Zweige, deren Wirkungskreise auch noch andere Organe zugehören.

Aus den verschiedenen Zählungen haben sich nun folgende Resultate ergeben:

Bei den Augenmuskeln des Schafes verhält sich die Anzahl der Nervenprimitivröhren zu den Muskelfasern wie folgt:

1) Näheres darüber s. im Anhang. 
Obliquus superior:

$\begin{array}{cc}\text { Nervenröhren, Muskelfasern. } & \text { Verhältniss. } \\ 2260: 8080 & 1: 3^{5} / 9 \\ 1972: 10790 & 1: 5^{1 / 2} \\ 2282: 11520 & 1: 5^{1 / 10} \\ 1098: 6589 & 1: 6 \\ 1756: 15385 & 1: 8^{1 / 3} \\ 1710: 16758 & 1: 9^{4} / 5 \\ \text { Obliquus inferior: } & 1: 3^{1 / 4} \\ 2374: 7620 & 1: 7^{1 / 3} \\ \text { Rectus inferior: } & 1: 4^{1 / 11} \\ 2002: 14716 & 1: 6^{1 / 6} \\ \text { Rectus medialis: } & 1: 6^{5} / 9 \\ 2002: 8705 & 1: 11^{1 / 3} \\ 3045: 18804 & 1: 11^{1 / 2} \\ 3662: 24010 & 1: 5^{1 / 2} \\ 2091: 23723 & 1: 10^{1 / 3} \\ 1640: 18860 & 1: 15 \\ 1920: 10624 & \text { Rectus lateralis: }\end{array}$

Der Rectus sup. ist übergangen, weil der zugehörige Nerv sich nicht ausschliesslich auf diesen Muskel beschränkt, sondern auch einige Zweige zum Levator palp. sup. abschickt, ein zuverlässiges Ergebniss für ihn also nicht zu erzielen war. Für die übrigen Augenmuskeln geht aber aus den obigen Angaben hervor, dass wirklich eine verhältnissmässig sehr bedeutende Anzahl von Nervenfasern in sie eintritt, wenngleich mehr oder weniger geringe Zahlenschwankungen, die in den Bereich der individuellen Verschiedenheiten fallen, natürlich nicht fehlen. Auch scheint sich aus vorstehenden Zahlen zu ergeben, dass unter ihnen der Obl. sup. und inf. am günstigsten situirt erscheinen, während umgekehrt bei dem Rect. lat. die wenigsten Nervenfasern vorhanden sind. Berechnet man aus den einzelnen Ziffern der Tabelle für jeden Muskel das Mittel, so ergeben sich auf eine Nervenröhre:

Für den Obl. inf. 3-4 Muskelfasern.

$" \begin{array}{ll}\text { Obl. sup. } 6-7 \\ \text { Rect. inf. } 7-8 \\ \text { Rect. med. } 8\end{array}$


Der Rect. med. also neigt sich schon mehr auf die Seite des Rect. lat., dagegen hält der Rect. inf. die Mitte zwischen sämmtlichen Augenmuskeln.

Dieselben Schliisse finden zum Theil auch auf die an menschlichen Augen gemachten Beobachtungen ihre Anwendung. Auch hier überwog die Zahl der Muskelfasern beim Rect. lat. die der Nervenprimitivröhren am meisten, so dass die letzteren zu den ersteren sich verhalten wie $3610: 11965\left(=1: 3^{1 / 4}\right)$, während der Rect. inf. das Verbältniss von 5206 Nerven zu 10351 M. (= $1: 2$ ) aufwies, und beim Rect. med. gar auf eine Nervenfaser noch keine zwei Muskelfasern kamen, indem sich hier 3300 Nervenröhren auf 5580 Muskelfäden vertheilten $\left(=1: 1^{38} / 55\right)$.

Bei einer Durchschnittsberechnung würden somit in einem Augenmuskel des Schafes im Mittel etwa 6--7 Muskelfasern auf eine Nervenprimitivröhre zu rechnen sein, während beim Menschen insofern aus den vorliegenden geringen Daten ein allgemein gültiger Schluss gezogen werden darf, ein noch weit überraschenderes Verhältniss zu Tage tritt, indem hier nicht mehr wie 7 Muskelfäden dem Einflusse von 3 Nervenröhren unterliegen.

Es liesse sich wohl schon a priori die Idee rechtfertigen, die auch schon Kühne (1. c.) andeutet, dass die Muskeln des wichtigsten Sinnesorganes vor allen andern Muskeln am glücklichsten in Betreff der Nervenzahl bestelle seien, und so finden wir es denn bei einem Vergleich mit anderen Muskeln auch wirklich bestätigt. Zählungen, die ich an verschiedenen Mm. biceps brachii und Sartorius junger Hunde vornahm, ergeben für den Biceps ein Mittel von $1: 83$, während beim Sartorius ca. 40-60 Muskelfasern auf eine Nervenfaser zu rechnen waren. Bei einem Biceps konnte ich sogar das Verhältniss von 1: 125 constatiren. Der Muskel von 52487 Fasern ') hatte hier über nicht mehr als 420 Nervenröhren zu verfügen.

Wie oben schon erwähnt, fand nun Reichert in dem Brusthautmuskel des Frosches, dass ein solcher mit 160 Fasern von 6-7 Nervenröhren versorgt wird, so dass sich also das Verhältniss wie 1:23-27 herausstellt. Auffallender Weise ist hiernach der Froschmuskel mit einer grösseren Anzahl von Nervenprimitivfasern versorgt, als der Biceps und Sartorius des Hundes, es geht also

1) Da hier die Fasern nicht ganz durchgehen, so ist die Zahlenbestimmung nur eine approximative. 
daraus der wichtige Satz hervor, dass die allgemeine Ansicht, welche den Muskeln der höheren Wirbelthiere ausnahmslos eine grössere Anzahl von Nerven zuschreibt, unrichtig ist. Vielmehr scheint nach meinen Untersuchungen lediglich die Function des Organes den Ansschlag zu geben, indem die äusserst fein einzustellenden Augenmuskeln die höchste, die weniger fein organisirten Extremitätenmuskeln dagegen eine geringere Anzahl von Nervenröhren führen. Es lässt sich dieser Satz leicht aus anderen Muskeln des Frosches beweisen. So fanden sich in dem ganz unwichtigen kleinen Abductor digiti V. pedis dieses Thieres 200 Muskelfasern, die von nur 5 Nervenröhren versorgt wurden, also ein Verhältniss wie $1: 40$, ähnlich dem Sartorius des Hundes. In dem für die Bewegung des Schwimmens wichtigen Sartorius des Frosches dagegen fanden sich für 460 Muskelfasern 28 eintretende Nervenfasern, es ergab sich also ein Verhältniss, wie $1: 16^{3} / 7$. Auch hier beim Frosch müssen, wenn der oben ausgesprochene Satz richtig ist, die Augenmuskeln eine Zahl von Nerven führen, die der bei höheren Thieren beobachteten gleichkommt, oder doch wenigstens nahe steht, und so fanden sich in der That folgende Zahlenwerthe:

$\begin{array}{ccc}\text { Nerve } & \text { Muskel } & \text { Verhältniss. } \\ 68 & 230 & \text { ca. } 1: 3 \\ 54 & 225 & \text { ca. } 1: 4 \\ 30 & 400 & \text { ca. } 1: 13 \\ 24 & 496 & \text { ca. } 1: 21\end{array}$

Es ergiebt sich daraus ein Verhältniss von $1: 10$, welches gewiss richtig ist, wenn man die unvollkommeneren Augenbewegungen des Frosches in Auschlag bringt.

Einen weiteren Beweis dass die Function des Muskels die Zahl der eintretenden Nervenröhren bestimmt, liefern ferner die Resultate, welche ich an den Schwanzmuskeln der Maus gewonnen habe, die deshalb gewählt wurden, weil in ihnen die Fasern die ganze Länge des Muskels durchziehen und also eine Querschnittszählung ein richtiges Resultat ergeben musste.

Zur Untersuchung derselben legte ich den unversehrten Schwanz, um ihn schnittiähig zu machen, einige Tage in Müllersche Flüssigkeit mit Salzsäure und machte dann nach vollendeter Härtung in absolutem Alkohol aufeinander folgende Querschnitte. In der grössten Dicke des Muskels zählte ich hierauf die Fasern 
und die Nervenröhren an der Stelle ihres Eintrittes. Vier derselben verhielten sich, wie folgt:

Von den beiden an der centralen Seite gelegenen Muskeln zählte der eine 232 Fasern, der zugehörige Nerv deren 11 (1:211/11) der andere 217, der Nerve 14 Fasern $\left(1: 15^{1 / 2}\right)$. Vor die beiden dorsal gelegenen Muskeln führte der eine mit 438 Fasern eipen Nerven von 11 Fasern $(1: 39 \%$, der gegenüber liegende von 458 Fasern war mit 12 Nervenröhren versorgt $\left(1: 38^{1} / 6\right)$. Im Durchschnitt würde danach auf 28-29 Muskel- eine Nervenprimitivfaser zu rechnen sein, was mit den Beobachtungen von Reichertüber den Brusthautmuskel des Frosches nahezu übereinstimmt.

Nach Darstellung dieser nummerischen Beziehungen zwischen Nerv und Muskel, wende ich mich noch mit einigen Worten zum Verlaufe des Nerven innerhalb des Muskels selbst. Um zu sehen, in welcher Weise sich der Nerv hier verhält, ob Theilungen stets nachweisbar seien, und $o b$ die Ausbreitung sich durch den ganzen Muskel erstreckt, oder wie Kölliker ${ }^{1}$ ) angibt, hauptsächlich gewisse Regionen einhalte, wurden die fast durchweg zur Untersuchung verwendeten frischen Augenmuskeln des Schafes erst einige Zeit in schwache Essigsäure gelegt und ihnen so eine grössere Durchsichtigkeit verliehen. Der Nerv durchzieht nun den Muskel in seiner ganzen Länge, und zwar bilden die Stammfasern namentlich in dem Bezirke der Eintrittsstelle, also im mittleren Drittel des Muskels grobe Plexus, deren Fasern sich nach kurzem Verlauf wieder zu kleinen, marklosen Plexus (Endplexus Valentin) vereinigen und nun Theilungen eingehen. Daher ist denn auch die Verfolgung einer Stammfuser in ihrer ganzen Länge mit den grössten Schwierigkeiten verknüpft, weil sie sich fast stets nach der Trennung vom Stamm sogleich einem vorbeilaufenden Bündel anschliesst, mit dem sie dann einen neuen Plexus eingeht.

Die Ausbreitung der letzteren geschieht nicht gleichmässig nach allen Seiten hin, sondern es finden sich die gröberen Geflechte hauptsächlich nur in der Gegend des Eintrittes, die feineren hier und im vorderen dem Bulbus zugekehrten Drittel. Das hintere Drittel dagegen ist nur sehr karg mit beiderlei Arten von Plexus bedacht, wie denn diese Partie auch weniger Endigungen zählt, als die ubrigen Regionen. Abgesehen jedoch von diesen Abweichungen

1) Handb. der Gewebelehre. 5. Aufl. p. 170. 
der einzelnen Partien unter sich, hat sich bei allen Augenmuskeln als übereinstimmendes Resultat der Untersuchung ergeben, dass der Nerv den Muskel vollständig durchsetzt, überall grobe und feinere Plexus bildet, Theilungen eingeht und das ganze Gebiet mit mehr oder weniger zahlreichen Nervenendigungen versorgt. Mit diesem letzteren Factum muss ich einer Behauptung von $K \ddot{u h n e}{ }^{1}$ ) entgegentreten, in der er die Erklärung abgibt, dass in jedem Muskel grosse Strecken vorkommen, wo keine Nerven anzutreffen seien, und dass namentlich die Enden in beträchtlicher Ausdehnung nervenfrei zu sein pflegen. Es hat dieser Ausspruch des genannten Antors jedenfalls keine allgemeine Berechtigung; sie mag wohl im Zusammenhalt mit Kölliker's Angaben, der den M. omohyoideus des Menschen untersucht hat, für lange und dünne Muskeln richtig sein, durchaus jedoch nicht für die von mir untersuchten Augenmuskeln.

Im grossen Ganzen geschieht nun die Theilung der Nerven auf zweierlei Weise, entweder innerhalb des Nervenstämmchens, oder ausserhalb desselben. Theilt sich die Faser noch während ihres Verlaufes im Stamm, was übrigens bei weitem seltener beobachtet wurde, als ausserhalb des letzteren, so hat dieselbe entweder mit dieser Theilung ihre Bahn vollendet, indem sie sich direct in Endigung verliert, oder es ist ihr, nachdem sie eine Endigung abgegeben, nach weiterem Verlaufe noch eine spätere Theilung vorbehalten, indem die erstere nur als eine Abzweigung betrachtet, ihre fernere Bahn in keiner Weise beeinträchtigt. So hatte ich Gelegenheit im Retractor bulbi der Katze ein Nervenstämmchen zu beobachten, in welchem zwei neben einander liegende Fasern dem gedachten Schema entsprachen. Die eine von diesen bog schon zeitig aus der Bahn des Stämmchens ab und verlor sich ohne weitere Theilung in zwei Endigungen, die bei einander lagen, die andere dagegen gab nicht weit davon unmittelbar im Bereich des Stammes einen Ast $a b$, der in eine Endplatte auslief, verfolgte aber dessenungeachtet in der Bahn des Stammes ihren eigentlichen Weg, bis sie in einem ganz anderen Distrikte neuen Theilungen unterlag, und mehrere Endigungen aufwies. Daraus erhellt schon von selbst, dass die Nervenfasern durchaus nicht auf eine oder auf mehrere nahe bei einander liegenden Muskelfasern beschränkt zu sein

1) Stricker's Handbuch l. c. 
brauchen, dass vielmehr selbst ganz entgegengesetzte Muskelfäden dem Impulse einer und derselben Nervenfaser unterworfen sein können.

Fast überall gibt sich die Theilung der Primitivfasern der Augenmuskel-Nerven als eine dichotomische zu erkennen, Dreitheilungen sind seltener und solche in 4, 5 oder noch mehr Zweige, wie sie Wagner in seinem Handwörterbuch von niederen Thieren abbildet, scheinen bei Säugethieren entweder gar nicht, oder doch jedenfalls sehr selten vorzukommen.

Die Zahl der jedesmaligen Theilungen, d. h. also die Summe der aus einer Nervenfaser schliesslich resultirenden Endplatten auch nur annähernd zu bestimmen, ist völlig unmöglich, wie aus Vorstehendem hervorgeht, doch lassen die sorgfältigen Untersuchungen Kühne's ${ }^{1}$ ) im Zusammenhalt mit meinen Beobachtungen wenigstens einen allgemeinen Schluss zu. Derselbe bearbeitete den Sartorius des Frosches und fand, dass jeder Muskelfaser wenigstens eine Nervenendigung zukommt, dass es aber viele gibt, welche mehr solche bis zu 6 und 8 enthalten. Nimmt man nun auf eine Muskelfaser durchschnittlich nur 2 Endigungen an, so ergibt sich, dass bei einer Zahl von 460 Muskelfasern und 28 Nervenröhren, jede dieser letzteren in mindestens 33 Endplatten zerfallen muss.

Auch in anderen Muskeln hat es ganz den Anschein, als ob sich die Sache in gleicher Weise verhielte, wenn man die vielen leicht zu beobachtenden Theilungen bedenkt, und es würden dann natürlich z. B. die einzelnen Fasern der Augenmuskeln eine grössere Zahl von Endigungen aufweisen, als die anderer Organe.

Herrn Dr. Merkel, unter dessen Leitung vorstehende Untersuchungen ausgeführt wurden, spreche ich zum Schlusse für seine freundlich gewährte Unterstützung meinen herzlichsten Dank aus.

\section{A $\mathbf{n} \mathbf{h} \mathbf{n}$ g.}

Der Fasertheilungen in den Augenmuskeln des Schafes wurde schon oben Erwähnnng gethan; sie wurden sehr vielfach beobachtet, und es schien deshalb interessant, dieselben näher zu untersuchen, da dergleichen bis jetzt nur an den Muskel-

1) Peripher. Endorgane. 
fasern des Herzens, der Zunge und in der Stammmuskulatur des Pferdes $\left.{ }^{1}\right)$ nachgewiesen wurden.

In der Regel erfolgt eine solche Theilung gegen das Ende der Faser, weit weniger häufig konnte ich eine Spaltung inmitten der Muskelfaser wahrnehmen. Dieselbe ist eine durchweg dichotomische, wenigstens ist mir niemals eine Drei- oder Mehrtheilung begegnet. Etwaigen Vermuthungen, dass die gedachten Theilungen sich als Frucht irgend einer mechanischen Verletzung aufklären möchten, wird schon von vorneherein durch die $\mathrm{K}$ ühne'sche Isolirungsmethode die Spitze gebrochen, indem bei ihrer Anwendung an beiden Aesten der Abzweigung das Sarcolemm nachweislich erhalten bleibt.

Hin und wieder habe ich auch einige sehr schöne und unzweifelhafte Anastomosen, wie sie Figg. 5 und 6 darstellen, beobachtet.

Die sämmtlichen Angaben von Biesiadecki und Herzig (l. c.) finden sich auch für die Augenmuskeln vollkommen bestätigt, so dass ich mich fast mit einem Hinweis auf die Figurentafel bescheiden kann.

In der Regel laufen die Muskelfasern beiderseits an den Enden kegelförmig zugespitzt aus, oder sie runden sich sowohl oben als unten stumpf $a b$; während in weniger häufigen Fällen auch zierliche, baumförmig verzweigte Gabelungen zur Beobachtung kommen. Ferner gehören Fasern, die an der einen Seite in lauter feine abgerundete Zacken oder kamm- und kegelförmige Spitzen endigen, nicht gerade zur Seltenheit. Ebenso kann eine Muskelfaser in ihrer ganzen Länge an der einen Seite kammartige oder mehr wellenförmige Erhöhungen zeigen (Fig. 1), oder diese zu kurzen Fortsätzen und Aestchen ausbilden (Fig. 2), welche selbst wieder einfach abgerundet oder mit Einschnitten an den Spitzen versehen sind. Einige Male sah ich die Muskelfaser auch mit einer Art von Anschwellung abschliessen, indem das eine oder andere Ende conisch aufgetrieben erschien.

In der schon mehrfach citirten Abhandlung von Biesi a decki und Herzig bringen dieselben auch die Frage über den Zusammenhang zwischen Muskel- und Sehnengewebe zur Sprache, indem

1) Biesiadecki und Herzig: Die verschiedenen Formen der quergestreiften Muskelfasern. Wien. Sitzungsb. 1858. 
sie die täuschenden Bilder des directen Ueberganges zwischen beiden daraus $\mathrm{zu}$ erklären suchen, dass bei solchen Trugbildern eine $\mathrm{Fa}$ ser nicht mit einem stumpfen Ende, sondern mit mehreren kegelförmigen Spitzen in das Sehnengewebe hineinrage. Für die Richtigkeit dieser Ansicht finden sich wohl niegends so schöne und zahlreiche Belege, als gerade in den Augenmuskeln. Ueberall sah ich hier die Endkegel und Ausläufer von den blassen Sehnenfäden umschlängelt, in einzelnen Fällen wurden selbst ziemlich weit vom eigentlichen Ende gelegen Spitzen von ihnen umstrickt (Fig. 4).

\section{Erklärung der Abbildnngen auf Taf. II.}

Sämmtliche Figuren stellen verschiedene Formen von verästelten, anastomosirenden und getheilten Muskelfasern aus den Augenmuskeln des Schafes dar.

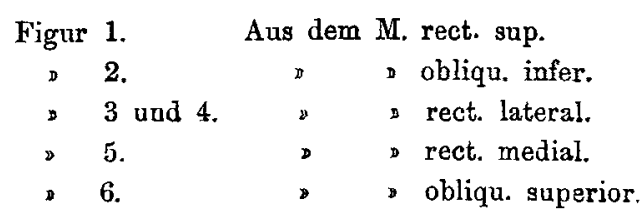




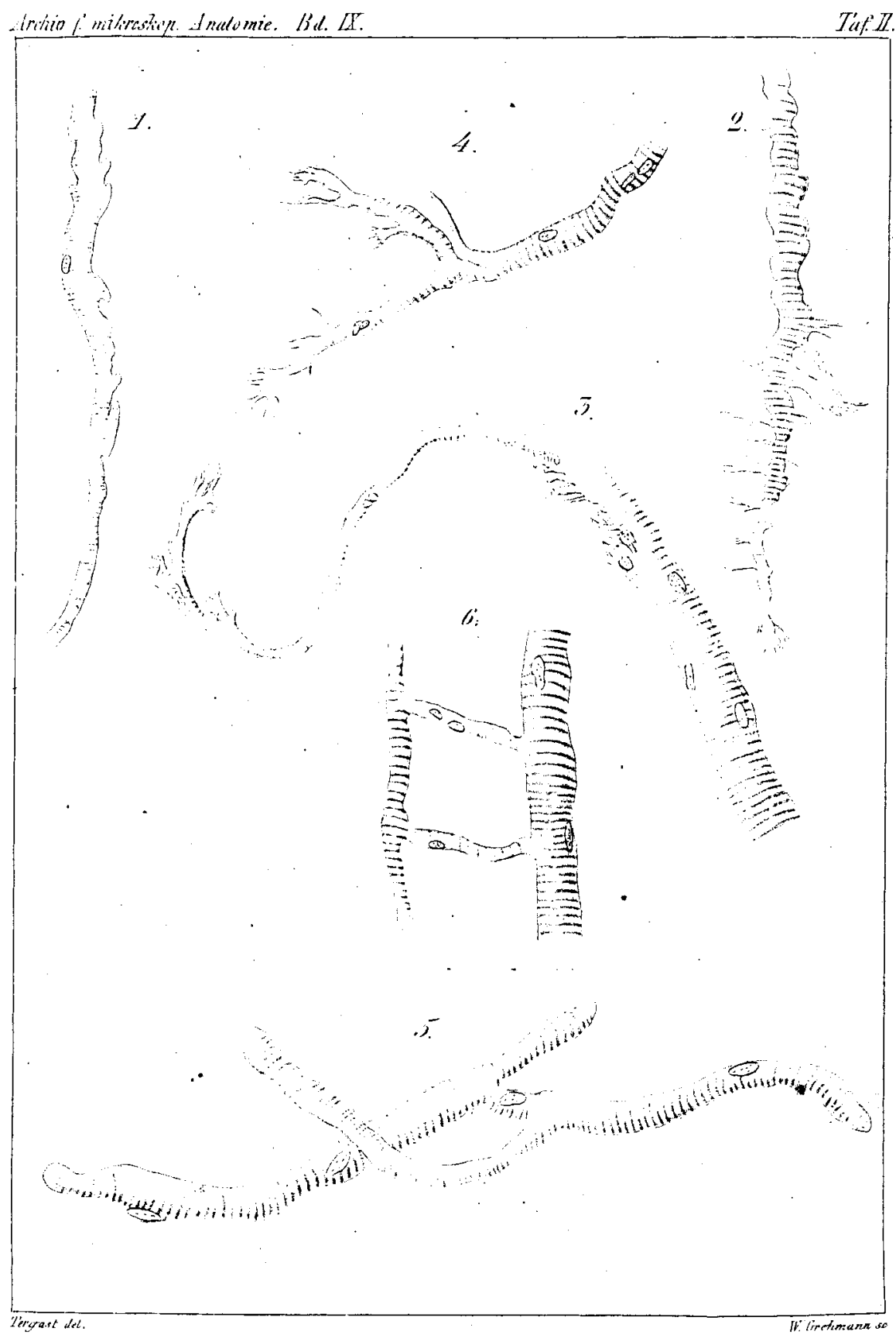

